\title{
How Can Sustainable Agriculture Increase Climate Resilience? A Systematic Review
}

\author{
Created by: QQDaniel El Chami
}

Version received: 21 April 2020

In the last few decades, a great deal has been written on the use of sustainable agriculture to improve the resilience of ecosystem services to climate change. However, no tangible and systematic evidence exists on how this agriculture would participate in alleviating impacts on vulnerable rural communities. This paper provides a narrative systematic review (SR) integrated with a bibliometric analysis and a concept network analysis to determine how, in this changing climate, sustainable agriculture can increase the resilience of agrosystems. Our search ranged from the date of the first relevant article until the end of 2018.

Sustainable agriculture is intended to increase the resilience of agrosystems to allow them to keep generating services even during extreme climate events ${ }^{[1][2]}$. To address this argument, this research has integrated multiple methods employing the ecosystem services framework adopted by the Millennium Ecosystem Assessment (MA), as described in the following sections.

\section{Bibliometric Analysis}

The Systematic Review (SR) protocol, which will be described in the next section, has been adopted for this analysis. The retrieved literature was exported into Mendeley (reference manager software). All the duplicate and spurious results were eliminated, and, before the relevance assessment, the bibliography was analyzed according to a list of indicators, including the source and type of documents, the year of publication, and the subject area. Bibliometric methods are now available in many scientific research methodologies $[3]$ because of the value they add in data interpretation.

\section{Concept Network Analysis}

This research also integrated a network analysis $\left.{ }^{4}\right][5][6]$, which has proven to be effective in text analysis. This method is based on a probabilistic assessment of word co-occurrence with relative word position, considering the structural properties of the text itself. This method graphically represents the network relationships between different keywords using qualitative and quantitative metrics[6].

The analysis was developed through different $\operatorname{step}^{[6]}$, beginning with the submission of the text to InfraNodus (https://infranodus.com/), a Web-based analytical engine. First, the text was prepared by removing frequent words to bind it together. The Krovetz Stemmer algorithm then stems the remaining words to reduce their redundancy and complexity ${ }^{[7][8]}$ and further normalizes the text before encoding. InfraNodus converts the submitted text into a network by performing a two-pass analysis. The graphical network can be used to represent the data visually using various software, such as Gephi for visualization and exploration ${ }^{[9]}$.

\section{Systematic Review Protocol}

The Systematic Review (SR) methodology is described in the systematic review guidelines developed by the Collaboration for Environmental Evidence as a way to inform practice and policy in environmental management $[10]$. The formulation of the primary question is the most important aspect in an SR, which (according to Pullin et al.[11]) requires a compromise between different approaches: a holistic approach and a reductionist one. Therefore, the primary research question that covers all the aspects of sustainable agriculture and its role in building resilience to climate change is the following: 
Following the systematic review conventions, this research question is broken down, as required by the Collaboration for Environmental Evidence (CEE) guidelines, into definable elements known as the PICO/PECO: a) the population (sustainable agriculture from the date of the first relevant publication onwards; however, this concept began to emerge in the $80 \mathrm{~s}^{[12]}$, so no location restrictions will be set because this concept could be applied to any agricultural system); b) interventions (interventions in the expansion of sustainable agriculture have been done since the 1980s, facilitating changes in natural and physical systems); c) comparators (sustainable agriculture versus conventional agriculture (Conventional agricultural systems are intensive mono-systems based on high input use.); and d) outcomes (resiliency parameters to climate change defined as the quantitative/qualitative changes in ecosystem services). The search terms used for this review were used to formulate different search keywords. A trial was undertaken on 18 September 2018 to refine the search terms using the Scopus database. One keyword was adopted for the systematic review after a discussion of the trial results with the co-authors and experts. This keyword was used to search the different scientific database sources, as well as the grey literature, which included only English publications.

All literature retrieved in the first step was screened for relevance according to the previously established inclusion criteria, which included a) relevant subjects (any country, any spatial scale); b) the type of intervention (sustainable management); c) comparators (which compare the benefits and/or impacts on natural and physical systems); d) methods (modeling, experiments, and surveys); e) outcomes (studies that consider the benefits/impacts on terrestrial and aquatic ecosystems, hydrology and water resource management, the natural environment, food and fiber production and security, immigration, poverty, human health, human infrastructure, and economic loss).

\section{Results}

Several observations have emerged from the results of this systematic review. Next, we discuss the observations and make some recommendations for the scientific community and policymakers in sustainable agriculture-related areas.

Sustainability is a three-dimensional model that requires a change in practice to contravene disciplinary boundaries, thereby realizing transdisciplinarity ${ }^{[13]}$, which represents a step forward for interdisciplinarity towards full integration. This goal is far from being achieved, according to Brandt ${ }^{[14]}$, and this review has further demonstrated that, in agriculture, we have not yet reached a level of interdisciplinarity where the knowledge and methods from different disciplines are unified into a synthetic approach.

The results confirm that different management aspects are commonly studied separately, which means that the literature has not studied sustainable agriculture as a whole but merely some practices and technologies that participate in sustainable agriculture, mainly through the improvement of system biodiversity and ecosystem services. To cope with the challenges of sustainable agriculture, changes in management strategies must be based on the integration of all management categories to produce a unique management method for agrosystems that accounts for soils, water, crops, genetics, the landscape, biodiversity, and knowledge and uses unified quantitative methods for evaluation and assessment.

The ecosystem-based approach described earlier in the paper is the most effective tool to achieve integration for the sustainable management of agrosystems under climate change because it is based on the management of all

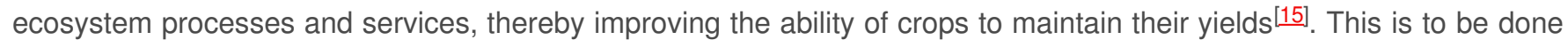
on a life cycle basis and has been successfully applied in the literature to agro-food systems (e.g., [16]) because it considers the interactions between resource use and potential impacts on biodiversity 17$]$. Finally, climate change impacts and resilience of agrosystems are highly dependent on local conditions (environmental, socio-economic, and management), which complicates the process of quantifying sustainable agriculture ${ }^{[18]}$.

\section{References}

1. Daniel Roberts; Autar K Mattoo; Sustainable Agriculture-Enhancing Environmental Benefits, Food Nutritional Quality and Building Crop Resilience to Abiotic and Biotic Stresses. Agriculture 2018, 8, 8, 10.3390/agriculture8010008.

2. Christine D. Barbeau; Maren Oelbermann; Jim D. Karagatzides; Leonard J S Tsuji; Sustainable Agriculture and Climate Change: 
Producing Potatoes (Solanum tuberosum L.) and Bush Beans (Phaseolus vulgaris L.) for Improved Food Security and Resilience in a Canadian Subarctic First Nations Community. Sustainability 2015, 7, 5664-5681, 10.3390/su7055664.

3. Ole Ellegaard; Johan A. Wallin; The bibliometric analysis of scholarly production: How great is the impact?. Scientometrics 2015, 105, 1809-1831, 10.1007/s11192-015-1645-z.

4. Roel Popping; Knowledge Graphs and Network Text Analysis. Social Science Information 2003, 42, 91-106, 10.1177/0539018403042001798.

5. Rafter Ferguson; Sarah Lovell; Permaculture for agroecology: design, movement, practice, and worldview. A review. Agronomy for Sustainable Development 2013, 34, 251-274, 10.1007/s13593-013-0181-6.

6. Paranyushkin, D. Identifying the Pathways for Meaning Circulation using Text. Network Analysis; Nodus Labs: Berlin, Germany, 2011. Available online: https://noduslabs.com/category/research/ (accessed in February 2019).

7. M.F. Porter; An algorithm for suffix stripping. Program 1980, 14, 130-137, 10.1108/eb046814.

8. Robert Krovetz; Viewing morphology as an inference process. Artificial Intelligence 2000, 118, 277-294, 10.1016/s00043702(99)00101-0.

9. Bastian, M.; Heymann, S.; Jacomy, M. Gephi: An open source software for exploring and manipulating networks. In Proceedings of the Third International AAAI Conference on Weblogs and Social Media, San Jose, CA, USA, 17-20 May 2009.

10. CEE. Guidelines for Systematic Review and Evidence Synthesis in Environmental Management; Version 5.0; Pullin, A.S., et al Eds.; Collaboration for Environmental Evidence (CEE): 2018. Available online: www.environmentalevidence.org/information-for-authors (accessed in February 2019 ).

11. Andrew Pullin; Teri M. Knight; Andrew R. Watkinson; Linking reductionist science and holistic policy using systematic reviews: unpacking environmental policy questions to construct an evidence-based framework. Journal of Applied Ecology 2009, 46, 970975, 10.1111/j.1365-2664.2009.01704.x.

12. Joyce Tait; E. Dick Morris; Sustainable development of agricultural systems: competing objectives and critical limits. Futures 2000, 32, 247-260, 10.1016/s0016-3287(99)00095-6.

13. Gertrude Hirsch Hadorn; David Bradley; Christian Pohl; Stephan Rist; Urs Wiesmann; Implications of transdisciplinarity for sustainability research. Ecological Economics 2006, 60, 119-128, 10.1016/j.ecolecon.2005.12.002.

14. Patric Brandt; Anna Ernst; Fabienne Gralla; Christopher Luederitz; Daniel J. Lang; Jens Newig; Florian Reinert; David James Abson; Henrik Von Wehrden; A review of transdisciplinary research in sustainability science. Ecological Economics 2013, 92, 1-15, 10.1016/j.ecolecon.2013.04.008.

15. Raffaele Vignola; Celia A. Harvey; Pável Bautista-Solís; Jacques Avelino; Bruno Rapidel; Camila Donatti; Ruth Martínez; Ecosystembased adaptation for smallholder farmers: Definitions, opportunities and constraints. Agriculture, Ecosystems \& Environment 2015, 211, 126-132, 10.1016/j.agee.2015.05.013.

16. Daniel El Chami; Andre Daccache; Assessing sustainability of winter wheat production under climate change scenarios in a humid climate - An integrated modelling framework. Agricultural Systems 2015, 140, 19-25, 10.1016/j.agsy.2015.08.008.

17. Gisela Lüscher; T. Nemecek; Michaela Arndorfer; Katalin Balázs; Peter Dennis; Wendy Jane Fjellstad; Juergen K. Friedel; Gérard Gaillard; Felix Herzog; Jean-Pierre Sarthou; et al.Siyka StoyanovaSebastian WolfrumPhilippe Jeanneret Biodiversity assessment in LCA: a validation at field and farm scale in eight European regions. The International Journal of Life Cycle Assessment 2017, 22, 1483-1492, 10.1007/s11367-017-1278-y.

18. Nadia El-Hage Scialabba; Maria Müller-Lindenlauf; Organic agriculture and climate change. Renewable Agriculture and Food Systems 2010, 25, 158-169, 10.1017/s1742170510000116.

\section{Keywords}

systematic review; sustainable agriculture; climate change; resilience; agrosystems

(C) 2020 by the author(s). Distribute under a Creative Commans CC BY license 